\title{
Application of Integral Equation Joined with the Chain Association Theory to Study Molecular Association in Sub- and Supercritical Water
}

\author{
Mohammad Nader Lotfollahi, ${ }^{\dagger}$ Hamid Modarress, ${ }^{\dagger}$ and G.Ali Mansoori $§, *$ \\ $(\dagger)$ Semnan University, Semnan, Iran, mnlotfollahi@semnan.ac.ir \\ (†)Amir-Kabir University of Technology,Tehran,Iran,hmodares@aut.ac.ir \\ (§) University of Illinois at Chicago, Chicago,IL,USA, mansoori@uic.edu
}

\begin{abstract}
:
In this report, the Percus-Yevick and the Ornestein-Zernike integral equations are solved simultaneously for the radial distribution functions of water at various state conditions, including sub- and supercritical states. The intermolecular potential function used in this study consists of an effective Kihara potential, which is derived for associated fluids. For derivation of the effective potential function, water is considered as a mixture of associated species due to hydrogen bonding. The contribution of hydrogen bonding is considered in the formulation of the effective Kihara potential parameters through the application of the analytic chain association theory. There is a good agreement between the present calculations and the experimental data in predicting the oxygen-oxygen radial distribution function near the critical point and at supercritical conditions for which experimental data are available. It is also concluded that at supercritical conditions a considerable degree of hydrogen bonding may be still present in the form of linear chain association. Therefore, the chain association model is valid near the critical point and at supercritical conditions instead of other structure models for the investigations on molecular structure of water.
\end{abstract}

\section{Introduction}

Exploring the microscopic structure of water has attracted the attention of many research workers. Water in sub- and supercritical conditions has been used as a solvent in separation processes or as a chemical media in several technological applications. Sub-critical water, on the basis of its selfassociation behavior, has many unusual properties. In supercritical conditions, physical properties are very sensitive to temperature and pressure, and near critical conditions, density and dielectric constant vary appreciably with pressure. Variation of rate constants of chemical reactions involving water with pressure at constant temperature is another important aspect of supercritical water. These peculiar characteristics of water are quite interesting for further studies.

There is an obstacle to the characterization of the behavior of water in terms of its hydrogen bonding structure and finding the effect of hydrogen bonding in its microstructure. Radial distribution function (RDF) represents the probability of finding a particle at a specified distance from an arbitrary central particle and is denoted by $g(r), r$ being the distance between particles. A variety of experimental data for water $g(r)$ are available in the literature, which are obtained by means of X-ray and neutron diffraction techniques. ${ }^{1-4}$ The Fourier transform of the $(g(r)-$ 1 ), which is generally named as the structure factor, ${ }^{5}$ has been measured for saturated liquid water in a wide temperature range of $298-473 \mathrm{~K}$ by Narten and Levy ${ }^{1}$ using an X-ray diffraction technique. The same technique has been used by Gorbatyi and Demyanets $^{2}$ to obtain the RDF of water in a wide temperature range of 298-773 K and at a pressure of 1000 bar. Soper et

(*) Corresponding author. al. $^{3,4}$ have reported the RDF of water from 298 to $673 \mathrm{~K}$ at various pressures, which includes both sub- and supercritical water states, using neutron diffraction technique.

The structures of water at sub-critical and at supercritical conditions have been studied numerically by computer simulation techniques using various intermolecular potential energy functions. ${ }^{6-12}$ According to these studies, the number of hydrogen bonds decreases as the temperature increases, and it is diminished asymptotically at very high temperatures. Some of the studies conclude that at supercritical conditions, almost all hydrogen bondings are broken down. Other studies $2,7,9$ indicate that some degree of hydrogen bonding at near critical and supercritical in the form of dimer and trimers is still present. Jedlovszky et al. ${ }^{10}$ have stated that the tetrahedral orientation of water is lost at high temperature, but hydrogen bonds are still present in the form of linear even at supercritical states.

One of the efficient procedures which is generally used for the RDF calculation is solving, simultaneously, the PercusYevick $(\mathrm{PY})^{13}$ and the Ornstein-Zernike $(\mathrm{OZ})^{14}$ integral equations for a given intermolecular potential energy function. Having a proper analytic potential function that can take the true intermolecular interactions and hydrogen bonding into account has always been the main barrier in application of integral equation to the water molecule RDF calculations. Touba et al. ${ }^{15}$ have shown that one can start with the Kihara ${ }^{16}$ potential function in order to develop an effective potential function for water molecules. They have introduced the contributions of hydrogen bonding into the parameters of the Kihara potential. To proceed with the calculations of the RDF, Touba et al. have reduced their proposed effective Kihara potential function to a simple applicable form, and then they have obtained the RDF only for the first shell of water molecules. Also in their 


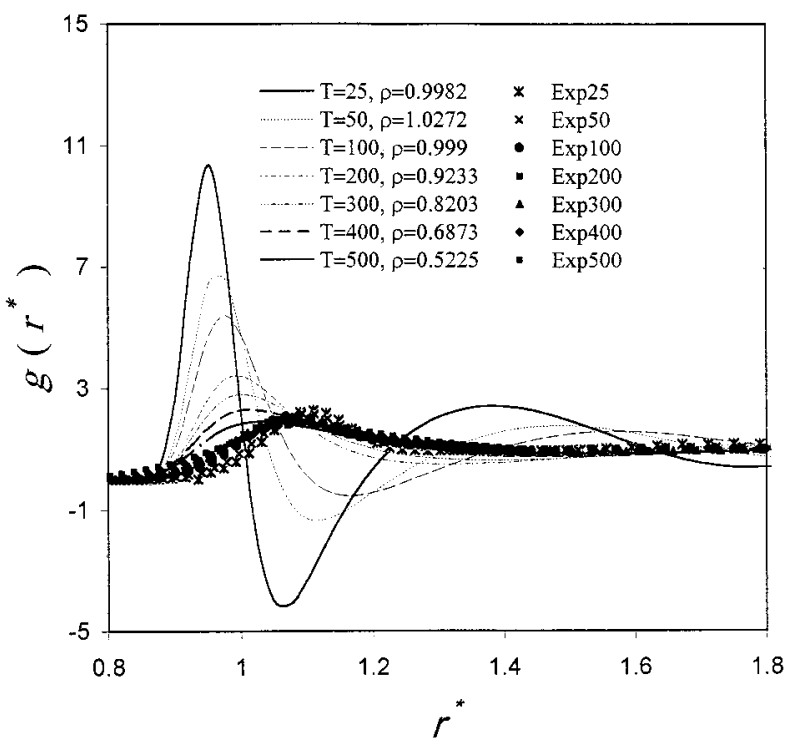

Figure 1. Comparison of calculated RDF using potential function in eq 1 with the experimental data for different temperatures and densities.

calculations, they ignored the temperature dependency of the intermolecular distances between the nearest neighboring molecules.

On the basis of the reported experimental data, ${ }^{2}$ it is evident that the intermolecular distances between the nearest neighbors are temperature-dependent. Then this temperature dependency should be accounted for in the potential function to be used in the RDF calculations. In this work, the complete form of the effective Kihara potential function, which includes the parameters for the temperature dependency of the nearest neighbors, is used to calculate the RDF (oxygen-oxygen radial distribution function) for water molecules. The method of RDF calculations in this work is based on the application of the integral equation, which, as the results obtained indicate, can be considered as an accurate approach and leads to evaluating the RDF in the whole range of intermolecular distance variations. Also, a new set of constants for the parameters of an effective Kihara potential function is proposed.

From a structural viewpoint, the results of these calculations substantiate the previous conclusion by the other workers ${ }^{2,7,9}$ that at supercritical conditions, hydrogen bonding in the form of linear chain associations exists between water molecules.

\section{Theory}

2.1. Potential Function. Calculation of the RDF by simultaneous solution of the Percus-Yevick (PY) and the OrnesteinZernike (OZ) integral equations requires having a proper functionality for $\phi(r)$, the pair intermolecular potential function. There are several equations for representing $\phi(r)$, which among them we can imply the following intermolecular potential function: ${ }^{17}$

$$
\phi(r)=4 \epsilon\left[\left(\frac{\sigma}{r}\right)^{12}-\left(\frac{\sigma}{r}\right)^{6}\right]-\frac{1}{r^{6}}\left(\frac{\mu^{4}}{3 k T}+2 \mu^{2} \alpha\right)
$$

In eq $1, \alpha$ is the polarizability factor, $\mu$ is the dipole moment, $\epsilon$ and $\sigma$ are energy and size parameters, and $r$ is the intermolecular separation. In this potential function, the dielectric property of pure water has been noted, and the dielectric dipole moment $\left(\mu^{4} / 3 k T\right)$ and induced dipole $\left(2 \mu^{2} \alpha\right)$ terms have been introduced in the intermolecular potential function. This equation is used in our RDF calculations. The results (Figure 1) indicate that this potential function cannot predict the RDF for water molecules. Therefore, a proper potential function should include parameters having flexibility to consider molecular associations between water molecules.

Blum and Vericat ${ }^{18,19}$ proposed a potential for the water intermolecular interaction consisting of hard sphere contribution, a point dipole, and a narrow attractive potential well with the symmetry of a tetrahedral octupole. As it is believed that the tetrahedral octupole structure of water molecule is not retained at supercritical conditions, this potential function is not considered in our calculations.

Another alternative form for potential function of water molecules is known as simple point charge potential (SPC), ${ }^{20}$ which considers the site-site interactions between water molecules as angle and charge dependent variables. This potential function is not used here, since it is not applicable to the molecular chain association.

The Kihara ${ }^{16}$ potential with a hard core distance parameter $\delta$, a soft-core distance parameter $\sigma$, and an energy parameter $\epsilon$ in the following form

$$
\phi(r)=4 \epsilon\left\{[(\sigma-\delta) /(r-\delta)]^{12}-[(\sigma-\delta) /(r-\delta)]^{6}\right\}
$$

is another alternative to represent the intermolecular potential function of water molecules. Of course, this intermolecular potential, in the above simple form, cannot consider molecular association of water molecules and fails to describe the peculiar behavior of water.

There is a bulk of evidence to prove that water can be considered as a mixture of different molecular species formed by molecular association due to hydrogen bonding. Association can be represented by reactions of the type $\mathrm{e}^{21-24}$

$$
\left(\mathrm{H}_{2} \mathrm{O}\right)_{i}+\left(\mathrm{H}_{2} \mathrm{O}\right)_{1} \stackrel{K_{i}(T)}{\longrightarrow}\left(\mathrm{H}_{2} \mathrm{O}\right)_{i+1} \quad i=1,2, \ldots, n
$$

The equilibrium constant of reaction 3 can be expressed as

$$
K_{i}=\left[x_{i+1} /\left(x_{i} x_{1}\right)\right]\left[\gamma_{i+1} /\left(\gamma_{i} \gamma_{1}\right)\right] \quad i=1,2, \ldots, n
$$

where $n$ is the limit of association and $x_{i}$ and $\gamma_{i}$ are the mole fraction and activity coefficient of $\left(\mathrm{H}_{2} \mathrm{O}\right)_{i}$, respectively. For simplicity, it is assumed that the associating species form an ideal solution and all $K_{i}$ 's are the same, then

$$
K=\left[x_{i+1} /\left(x_{i} x_{1}\right)\right]
$$

Equation 4 can be extended to different $i$ 's as follows:

$$
\begin{gathered}
x_{2}=K x_{1}{ }^{2} \\
x_{3}=K x_{2} x_{1}=K^{2} x_{1}{ }^{3} \\
x_{4}=K x_{3} x_{1}=K^{3} x_{1}{ }^{4} \\
: \\
: \\
x_{i+1}=K x_{i} x_{1}=K^{i} x_{1}{ }^{i+1}
\end{gathered}
$$

Since summation of all mole fractions is unity, then

$$
\sum_{i=1}^{n} K^{i} x_{1}^{i+1}=1
$$

It may be shown that the above series converge to 


$$
x_{1}\left[1-\left(K x_{1}\right)^{n}\right] /\left(1-K x_{1}\right)=1
$$

Considering $K x_{1}<1$ gives

$$
x_{1} /\left(1-K x_{1}\right)=1
$$

For an associating fluid having a large number of associating components, compositions may be replaced with a composition distribution function $\chi(I)$, where $I$ is the number of associated monomers. ${ }^{25}$ For the case where $n$ is very large, the summation in eq 7 can be replaced with an integral

$$
\int_{0}^{n} \chi(I) \mathrm{d} I=1
$$

where $\chi(I)$ is defined as follows:

$$
\chi(I)=\chi_{\mathrm{o}} K^{I} x_{1}{ }^{I+1}
$$

$\chi_{\mathrm{o}}$ is the normalized factor and can be calculated using eq 10

$$
\chi_{\mathrm{o}}=-\ln \left(K x_{1}\right) / x_{1}
$$

Substituting $\chi_{\mathrm{o}}$ and $x_{1}$ in eq 11 gives

$$
\chi(I)=-[K /(K+1)]^{I} \ln [K /(K+1)]
$$

Using the conformal solution theory, which assumes that there exists a pure hypothetical fluid with the same properties as those of the mixture at the same density and temperature, $\Phi_{i j}$ can be represented as follows:

$$
\Phi_{i j}=\epsilon_{i j} \Phi_{\mathrm{oo}}\left(r / \sigma_{i j}\right)
$$

where subscript (oo) denotes the reference fluid and the parameters $\sigma_{i j}$ and $\epsilon_{i j}$ represent the molecular conformal energy and volume parameter, respectively. The mixture theory is considered very important in extending the applicability from pure fluids to fluid mixtures.

Among the statistical mechanical conformal solution theories of mixtures, one-fluid van der Waals theory is simple to use and accurate enough with the following form:

$$
\sigma^{3}=\sum_{i} \sum_{j} x_{i} x_{j} \sigma_{i j}^{3}
$$

and

$$
\epsilon \sigma^{3}=\sum_{i} \sum_{j} x_{i} x_{j} \epsilon_{i j} \sigma_{i j}^{3}
$$

Applying a similar mixing rule as that $\sigma$ of to core diameter $\delta$ in Kihara potential function gives

$$
\delta^{3}=\sum_{i} \sum_{j} x_{i} x_{j} \delta_{i j}^{3}
$$

Therefore, the parameters of the Kihara potential $(\sigma, \delta$, and $\epsilon)$ are expressed in terms of parameters for various species $\left(\sigma_{i j}\right.$, $\delta_{i j}$, and $\epsilon_{i j}$ ) formed due to hydrogen bonding among water molecules. Applying the combining rules $\sigma_{i j}{ }^{3}=\left(\sigma_{i}^{3}+\sigma_{j}^{3}\right) / 2$, $\delta_{i j}{ }^{3}=\left(\delta_{i}{ }^{3}+\delta_{j}{ }^{3}\right) / 2$, and $\epsilon_{i j}=\left(\epsilon_{i} \epsilon_{j}\right)^{1 / 2}$ and for a mixture, the Kihara potential parameters $(\sigma, \delta$, and $\epsilon)$ can be expressed by the well-known van der Waals mixing rule: ${ }^{15}$

$$
\begin{gathered}
\sigma^{3}=\sum_{i} \sum_{j} x_{i} x_{j}\left[\left(\sigma_{i}^{3}+\sigma_{j}^{3}\right) / 2\right]=\sum_{i} x_{i} \sigma_{i}^{3} \\
\delta^{3}=\sum_{i} \sum_{j} x_{i} x_{j}\left[\left(\delta_{i}^{3}+\delta_{j}^{3}\right) / 2\right]=\sum_{i} x_{i} \delta_{i}^{3} \\
\epsilon \sigma^{3}=\sum_{i} \sum_{j} x_{i} x_{j}\left(\epsilon_{i} \epsilon_{j}\right)^{1 / 2}\left[\left(\sigma_{i}^{3}+\sigma_{j}^{3}\right) / 2\right]= \\
\sum_{i}\left(x_{i} \epsilon_{i}^{1 / 2} \sigma_{i}^{3}\right) \sum_{j} x_{j} \epsilon_{j}^{1 / 2}
\end{gathered}
$$

where $x$ represents mole fraction and $i$ and $j$ refer to species in the mixture.

The type of species formed in water due to molecular association allows one to assume that there is a continuous molecular weight distribution of various species and therefore eqs 18,19 , and 20 can be written in the following form: ${ }^{15}$

$$
\begin{gathered}
\sigma^{3}=\int_{0}^{\infty} \chi(I)[\sigma(I)]^{3} \mathrm{~d} I \\
\delta^{3}=\int_{0}^{\infty} \chi(I)[\delta(I)]^{3} \mathrm{~d} I \\
\epsilon \sigma^{3}=\int_{0}^{\infty} \chi(I)[\epsilon(I)]^{1 / 2}[\sigma(I)]^{3} \mathrm{~d} I \int_{0}^{\infty} \chi(I)[\epsilon(I)]^{1 / 2} \mathrm{~d} I
\end{gathered}
$$

The parameters $[\sigma(I)]^{3},[\delta(I)]^{3}$, and $[\epsilon(I)]^{1 / 2}$ are selected to be the function of distribution index " $P$ "

$$
\begin{gathered}
{[\sigma(I)]^{3}=\sigma_{1}{ }^{3} I_{1}^{\zeta}} \\
{[\delta(I)]^{3}=\delta_{1}{ }^{3} I_{2}^{\zeta}} \\
{[\epsilon(I)]^{1 / 2}=\epsilon_{1}^{1 / 2}\left[\zeta_{5} I_{3}^{\zeta}+\left(1-\zeta_{5}\right) I_{4}^{\zeta}\right]}
\end{gathered}
$$

Integration of the above equations leads to

$$
\begin{gathered}
\sigma=\sigma_{1}\left[\Gamma\left(1+\zeta_{1}\right) \theta_{1}^{\zeta_{1}}\right]^{1 / 3} \\
\delta=\delta_{1}\left[\Gamma\left(1+\xi_{2}\right) \theta_{2}^{\zeta_{2}}\right]^{1 / 3} \\
\epsilon=\epsilon_{1} \theta^{2 \zeta_{3}}\left[\Gamma\left(1+\zeta_{1}+\zeta_{3}\right) \Gamma\left(1+\zeta_{3}\right) / \Gamma\left(1+\zeta_{1}\right)\right]\left\{1+\zeta_{3} /\right. \\
\left.\left[\left(\zeta_{1}+\zeta_{3}\right) \theta\right]-\zeta_{3}+\zeta_{4}\left[1-\left(2+\zeta_{1}+\zeta_{3}\right) \theta\right]\right\}\{1+1 / \\
\left.\theta-\zeta_{3}+\zeta_{4}\left[1-\left(2+\zeta_{3}\right) \theta\right]\right\}
\end{gathered}
$$

In the above equations, $\sigma_{1}, \delta_{1}$, and $\epsilon_{1}$ are the potential energy parameters for a water monomer, $\zeta_{1}, \zeta_{2}, \zeta_{3}$, and $\zeta_{4}$ are constants, $\Gamma$ is the gamma function, and $\theta$ can be expressed in terms of equilibrium constant, $K(T)$, of the hydrogen bonding reaction

$$
\theta=-1 / \ln [K(T) /(1+K(T))]
$$

The equilibrium constant of the hydrogen bonding reaction can be expressed as

$$
\ln K(T)=\left(T \Delta S^{\circ}-\Delta H^{\circ}\right) / R T
$$

The values of $\Delta S^{\circ}$ and $\Delta H^{\circ}$ for the above reactions can be obtained from the experimental Raman spectroscopic measurement. ${ }^{26}$

In the present work, the complete set of the parameters for the effective Kihara potential function (eqs 27-29) have been used for the RDF calculations of water molecules by the integral equation method and the constants of these parameters have been evaluated.

2.2. Integral Equation. The radial distribution function (RDF) at a distance $r$ can be expressed in terms of the total correlation function, $h\left(r^{*}\right)$, as 


$$
g\left(r^{*}\right)=h\left(r^{*}\right)+1
$$

where $r^{*}=r / \sigma$. To obtain the RDF, the $\mathrm{OZ}$ along with the PY equations is solved simultaneously. The PY equation for a onecomponent system interacting through the pair potential $\phi\left(r^{*}\right)$ can be written as ${ }^{13}$

$$
c\left(r^{*}\right)=\left[1+\gamma\left(r^{*}\right)\right]\left(\exp \left(-\beta \phi\left(r^{*}\right)\right)-1\right)
$$

where $\beta \equiv 1 / k T$ and

$$
\gamma\left(r^{*}\right)=h\left(r^{*}\right)-c\left(r^{*}\right)
$$

In the above equation, $\gamma\left(r^{*}\right)$ is the indirect correlation function, and $h\left(r^{*}\right)$ is the total correlation function. The direct correlation function $c\left(r^{*}\right)$ is defined in terms of $h\left(r^{*}\right)$ by the $\mathrm{OZ}$ equation as $^{14}$

$$
h\left(r^{*}\right)-c\left(r^{*}\right)=\rho \int c\left(\left|r^{*}-r^{*}\right|\right) h\left(r^{\prime *}\right) \mathrm{d} r^{*}
$$

where $\rho$ is the particle number density.

To solve for $g(r)$ numerically, one has to express the PY equation in a discrete form as

$$
c_{i}=\left(1+\gamma_{i}\right)\left[\exp \left(-\beta \phi_{i}\right)-1\right]
$$

The discrete Fourier transform of $c_{i}$ can be defined as

$$
\tilde{\mathrm{c}}_{j}=\left(4 \pi \delta r / k_{j}\right) \sum_{i=1}^{1 / 2 N-1} r_{i} \sin k_{j} r_{i} c_{i}
$$

where $k_{j}=2 \pi j / N \delta r^{*}, N$ is the number of division, and $\delta r^{*}$ is the interval distance of divisions. Similarly, by Fourier transform, the $\mathrm{OZ}$ equation can be expressed as

$$
\tilde{\gamma}_{j}=\rho \tilde{\mathrm{c}}_{j}^{2} /\left(1-\rho \tilde{\mathrm{c}}_{j}\right)
$$

where $\tilde{\gamma}_{j}$ is the Fourier transform of the indirect correlation function. For numerical calculation, Gillan 's method, which is rapidly convergent, is used. ${ }^{27}$ This method is a combination of the traditional iterative scheme and a Newton-Raphson technique. In what follows, we perform a number of calculations using the proposed method, and the results are compared with the experimental RDF data of water.

\section{Results and Discussion}

A complete theory for intermolecular interactions of water molecules is still to be developed; therefore, some of the investigators satisfied themselves with partial theories involving some aspects of water molecules. Selection of these theories depends on which properties of water are to be focused on. We used eq 1 for RDF calculations for water molecules. In Figure 1 , the results of calculations based on eq 1 are compared with the experimental data obtained by Gorbatyi and Demyanets. ${ }^{2}$ The large deviations between the calculated RDF and experimental data indicate that the potential function expressed by eq 1 is not adequate for describing the molecular associations due to hydrogen bonding of water molecules, since this equation considers only dielectric properties of water molecules.

Equation 2 is another alternative for calculations of the water RDF using the integral equation method. This equation in principle consists of an effective Kihara potential function whose parameters are temperature-dependent. This potential function, as it was explained before, takes the hydrogen bonding into account according to the chain association theory and the

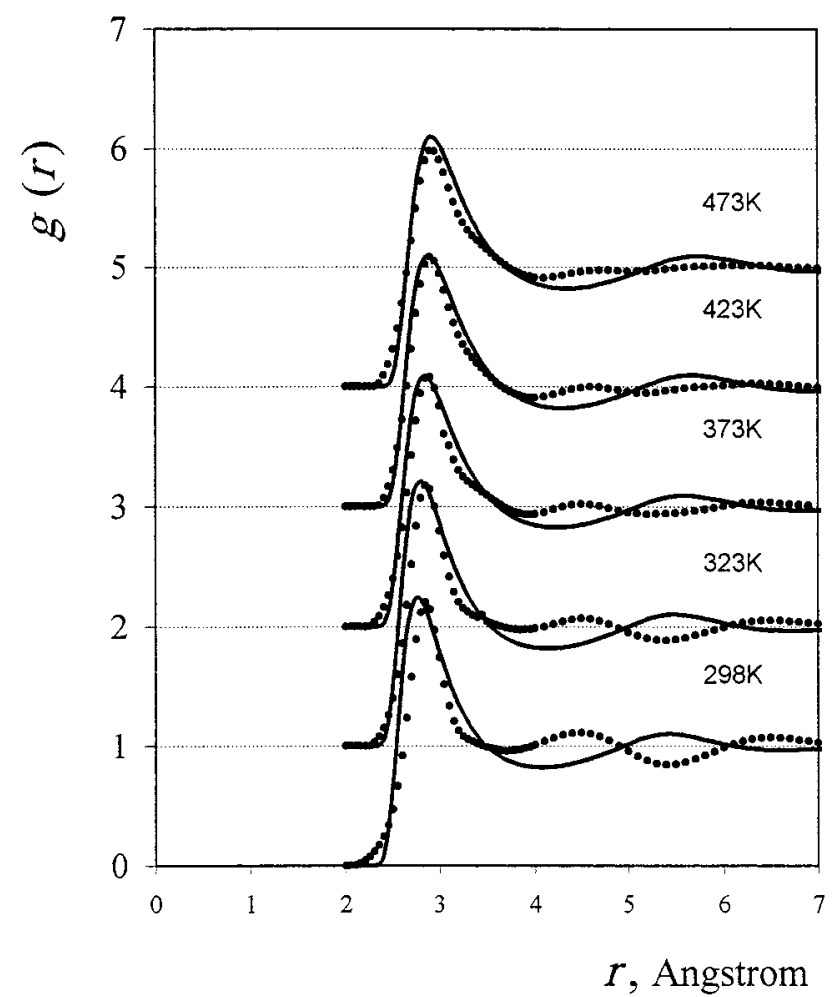

Figure 2. Comparison of RDF calculated by the present approach and the experimental RDF data $^{2}$ at $P=1000$ bar and for different temperatures.

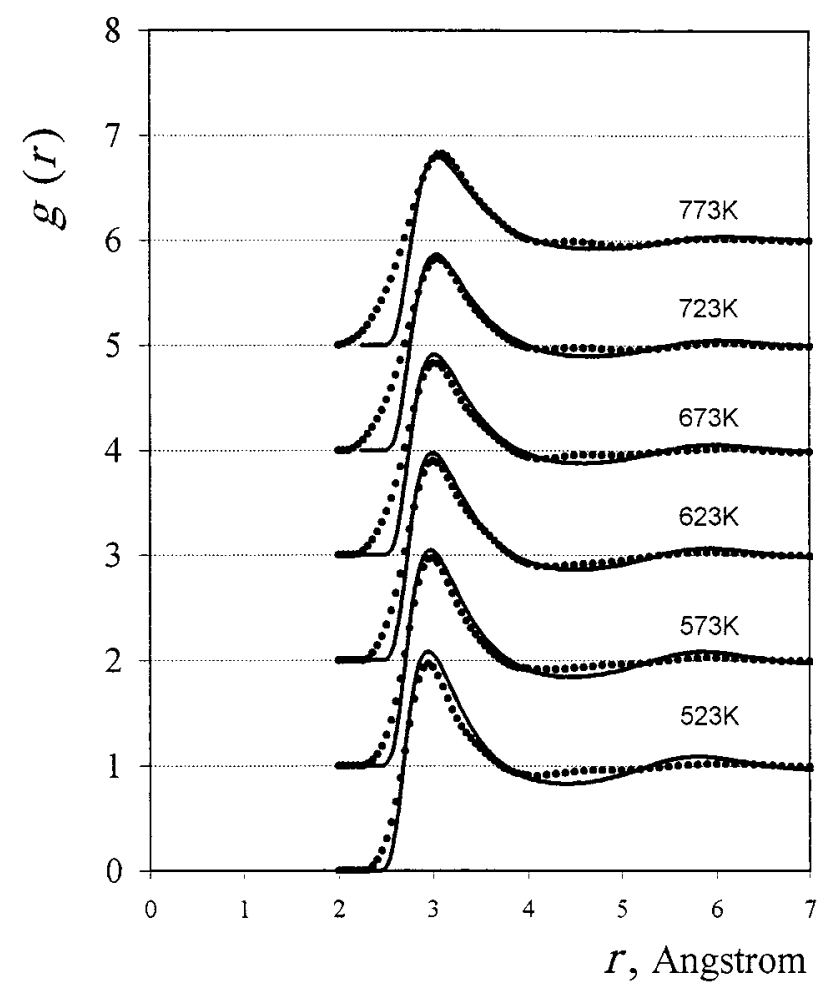

Figure 3. Comparison of RDF calculated by the present approach and the experimental RDF data $^{2}$ at $P=1000$ bar and for different temperatures.

conformal solution theory. In this way, the effect of hydrogen bonding is introduced in a realistic way in the parameters of the Kihara potential.

Numerical values of the constants of the proposed effective Kihara potential energy function $\left(\sigma_{1}, \delta_{1}, \epsilon_{1}, \zeta_{1}, \zeta_{2}, \zeta_{3}, \zeta_{4}\right)$ are not available. For calculating these constants, a set of the RDF 


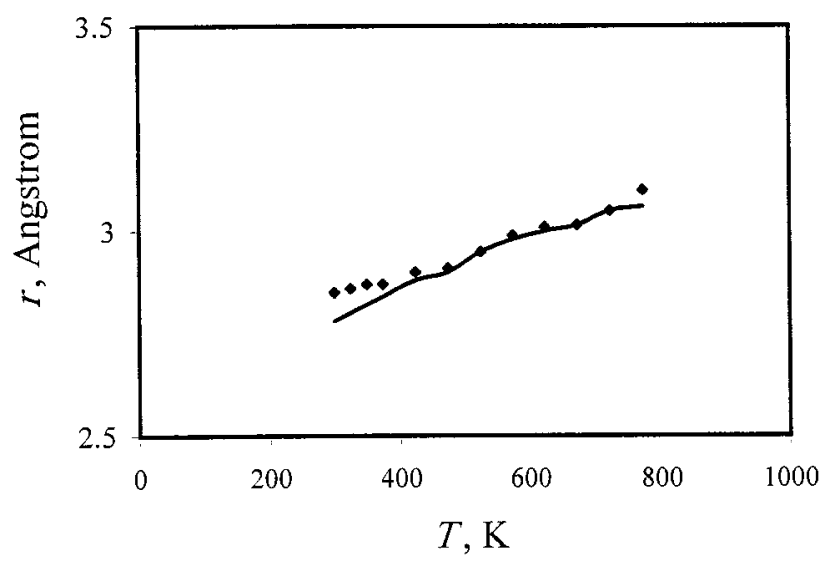

Figure 4. Temperature dependence of the intermolecular distance between the nearest neighbors; line represents the results by present approach, and points represent the results of experimental data. ${ }^{2}$

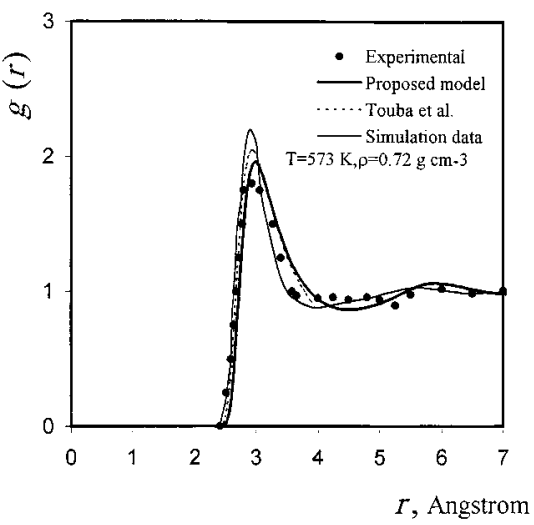

Figure 5. Comparison of RDF calculated by the present approach, Touba et al. ${ }^{15}$ method, computer simulation results, ${ }^{11}$ and the experimental data of water in near critical point ${ }^{4}$ at $T=573 \mathrm{~K}$ and $\rho=0.72$ $\mathrm{g} \mathrm{cm}^{-3}$.

data $^{2}$ at various temperatures is used, and by the application of a numerical regression technique, the constants are adjusted so that the RDF data will match the predictions by the integral equation. The calculated parameters for water $\left(\xi_{1}, \zeta_{2}, \zeta_{3}\right.$, and $\left.\zeta_{4}\right)$ are $-0.07,0.1,0.48$, and 0.0036 , and the potential energy parameters for the water monomer $\left(\sigma_{1}, \delta_{1}\right.$, and $\left.\epsilon_{1} / k\right)$ are 2.695 $\AA$, $0.35 \AA$, and $110 \mathrm{~K}$, respectively.

Figures 2 and 3 show the calculated RDFs by the effective Kihara potential function using the present method along with the experimental RDF data ${ }^{2}$ for water at $(298,323,373,423$, 473,523, 573, 623, 673, 723, and 773) $\mathrm{K}$ and 1000 bar pressure. These data are for liquid water $(298-623 \mathrm{~K})$ and for water at the supercritical condition $(673-773 \mathrm{~K})$. The calculated values in these figures have been obtained by solving the integral equation using the effective Kihara potential. According to these figures, the proposed model predicts the first shell of the water RDF quite accurately for all the temperatures. However, prediction of the location of the second shell is improved as the temperature increases. It is interesting to note that the position of the maximum of the first shell in Figures 2 and 3 is temperature-dependent. The intermolecular distance between the nearest neighbors at $298 \mathrm{~K}$ is at about $2.85 \AA$, and it increases gradually to $3.1 \AA$ at $773 \mathrm{~K}$. The calculation results show that the presented model is able to predict this effect quite accurately. (Figure 4)

Figures 5 and 6 are for RDF at conditions $T=573 \mathrm{~K}, \rho=$ $0.72 \mathrm{~g} \mathrm{~cm}^{-3}$, and $T=673 \mathrm{~K}, \rho=0.66 \mathrm{~g} \mathrm{~cm}^{-3}$. In these conditions, water is near its critical point and in the supercritical

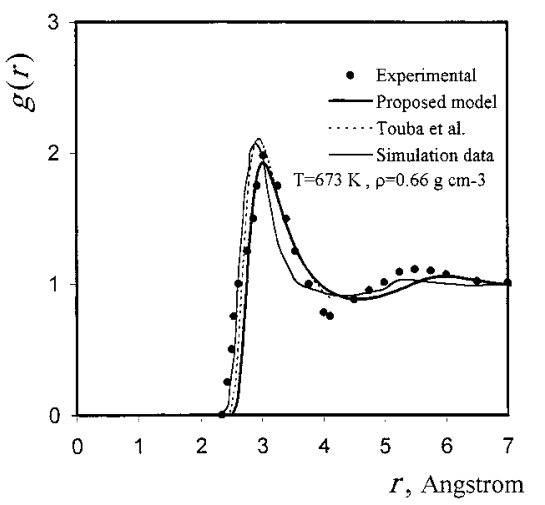

Figure 6. Comparison of RDF calculated by the present approach, Touba et al. ${ }^{15}$ method, computer simulation results, ${ }^{11}$ and the experimental data of water in supercritical condition ${ }^{4}$ at $T=673 \mathrm{~K}, \rho=$ $0.66 \mathrm{~g} \mathrm{~cm}^{-3}$.

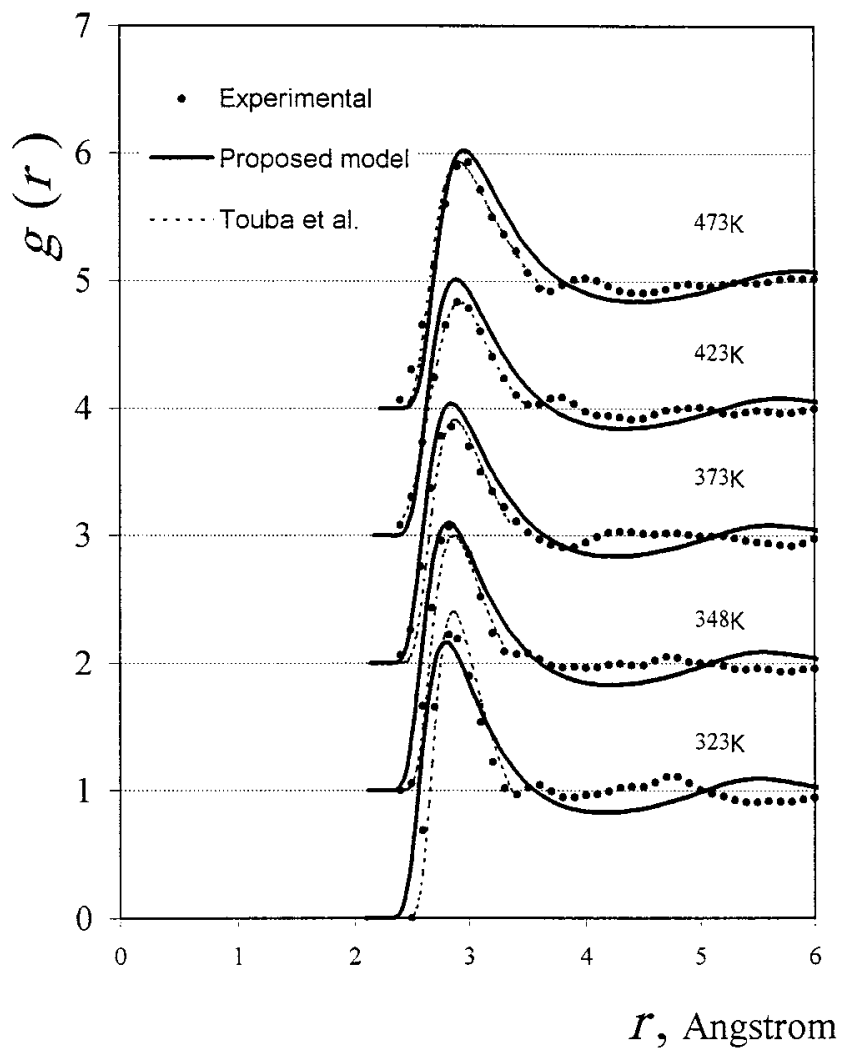

Figure 7. Comparison of RDF calculated by the present approach, the method due to Touba et al., ${ }^{15}$ and the experimental data ${ }^{1}$ at various temperatures for saturated water.

state, respectively. In these figures, the results of the calculations are compared with the model due to Touba et al., ${ }^{15}$ the computer simulation data ${ }^{11}$, and experimental data. ${ }^{4}$ In their calculations, Touba et al. made a simplifying assumption that $\zeta_{1}=0, \zeta_{2}=$ 1. Taking $\zeta_{1}=0$ eliminates the temperature dependency of the parameter $\sigma$ in the Kihara potential function, which, in contrast to the experimental evidence, ${ }^{2}$ requires that the intermolecular distances between the nearest neighboring molecules is not dependent on temperature. Their RDF calculations for water molecules were done by an analytical expression originally proposed by Touba and Mansoori ${ }^{28}$ and was able to predict the first shell only.

According to Figures 5 and 6 , the calculations by the proposed model are in good agreement with the experimental data.

Figure 7 compares the RDF obtained from the integral equation, the experimental data for saturated water, ${ }^{1}$ and the 
results of Touba et al. ${ }^{15}$ for the first shell of RDF of water molecule. It is seen that the calculated RDF based on the present model agrees with both the experimental data and the Touba et al. results for the first shell of RDF.

Finally, considering the results presented in Figures $1-7$, it is seen that the agreement of the present model with experimental data is good, especially for prediction of the first shell of the RDF and this agreement improves with increasing temperature. The calculations presented here indicate that a considerable degree of hydrogen bonding remains at supercritical conditions. This observation is consistent with the opinion of other authors. ${ }^{7,9,10}$ Jedlevszky et al. ${ }^{10}$ state that the tetrahedral orientation of the hydrogen bonded neighbors is already lost at $423 \mathrm{~K}$, whereas the hydrogen bonds themselves remain preferentially linear even above critical point. Kalinichev ${ }^{9}$ concluded that some degree of hydrogen bonding at the highest temperature is still present in the form of dimers and trimers. Dang ${ }^{7}$ indicated that a considerable degree of hydrogen bonding remains at near critical conditions. Therefore, chain association theory which is consistent with the linear form of structure for hydrogen bonds is valid near the critical point and at supercritical conditions instead of other forms of the assumed structures for water molecules.

In comparing the present calculations with the experimental RDF data of water, one can conclude that the integral equation method, joined with the effective pair potential based on the chemical association theory, is able to predict of water RDF at near critical and supercritical conditions. Therefore, once again, it is worthwhile to emphasize that the calculated RDFs for water substantiates the conclusion made by other authors ${ }^{7,9,10}$ that at these conditions the water molecules associate in the form of linear chains and not in the form of networks.

Acknowledgment. We would like to thank Dr. H. Touba for his useful discussion.

\section{References and Notes}

(1) Narten, A. H.; Levy, H. A. J. Chem. Phys. 1971, 55, 5, 2263.

(2) Gorbatyi, Yu. E.; Demyanets, Yu. N. J. Struct. Chem. 1983, 24, 385.

(3) Postorino, P.; Tromp, R. H.; Ricci, M. A.; Soper, A. K.; Nellson, G. W. Nature 1993, 366, 69. 247.

(4) Soper, A. K.; Bruni, F.; Ricci, M. A. J. Chem. Phys. 1997, 106, 1,

(5) McQuarrie, D. A. Statistical Thermodynamics, 1st ed.; Harper \& Row Publishers Inc.: New York, 1976.

(6) Yoshii, N.; Yoshie, H.; Miura, S.; Okazaki, S. J. Chem. Phys. 1998 110, 12, 4873.

(7) Dang, L. X. J. Phys. Chem. B 1998, 102, 620.

(8) Ortega, J.; Lewis, J. P.; Sankey, O. T. J. Chem. Phys. 1997, 106, 9,3696 .

(9) Kalinichev, A. G.; Bass, J. D. J. Phys. Chem. A 1997, 101, 9720.

(10) Jedlovszky, P.; Brodholt, J. P.; Bruni, F.; Ricci, M. A.; Soper, A K.; Vallauri, R. J. Chem. Phys. 1998, 108, 20, 8528.

(11) Chialvo, A. A.; Cummings, P. T. J. Chem. Phys. 1996, 100, 1903.

(12) Blum, L.; Degreve, L. Mol. Phys. 1996, 88, 2, 585-590.

(13) Percus, J. K.; Yevick, G. J. Phys. Rev. 1958, 110, 1.

(14) Ornstein, L. S.; Zernike, F. Proc. Akad. Sci. (Amsterdam) 1914 $17,793$.

(15) Touba, H.; Mansoori, G. A.; Matteoli, E. Int. J. Thermophys. 1998, 19 (5), 1447-1471.

(16) Kihara, T. Rev. Mod. Phys. 1953, 25, 831.

(17) Reed, T. M.; Gubbins, K. E. Applied Statistical Mechanics; McGraw-Hill Book Company: New York, 1973; p 161.

(18) Blum, L.; Vericat, F. Mol. Phys. 1995, 86, 4, 809-817.

(19) Blum, L.; Vericat, F. J. Phys. Chem. 1996, 100, 1197-1205.

(20) Berendson, H. J. C.; Postma, J. P. M.; von Gunsteren, W. F.; Hermans, J. Intermolecular Forces; Proceeding of the Fourteenth Jerusalem Symposium on Quantum Chemistry and Biochemistry; Pullman, C., Ed.; Reidel: Dordrecht, The Netherlands, 1981; p 331.

(21) Anderko, A. Fluid Phase Equilib. 1992, 75, 89

(22) Campbell, S. W. Fluid Phase Equilib. 1994, 102, 61.

(23) Aghamiri, S. F.; Mansoori, G. A.; Modarress, H. Z. Phys. Chem. Board 1998, 205, 211.

(24) Aghamiri, S. F.; Mansoori, G. A.; Modarress, H. J. Phys. Chem. B 2001, (In Press).

(25) Al-Mutawa, A. H.; Hwang, C. A.; Kim, S. T.; Mansoori, G. A. Trends Chem. Eng. 1993, 1, 175.

(26) Walrafen, G. E.; Fisher, M. R.; Hokmabadi, M. S.; Yang, W. H. J. Chem. Phys. 1986, 85, 6970.

(27) Gillan, M. J. Mol. Phys. 1979, 38, 6, 1781.

(28) Touba, H.; Mansoori, G. A. Fluid Phase Equilib. 1996, 119, 51. 